\title{
Electroplating of Non-Fluorinated Superhydrophobic Ni/WC/WS 2 Composite Coatings with High Abrasive Resistance
}

Jixue Zhou ${ }^{\mathrm{a}, *}$, Guochen Zhao ${ }^{\mathrm{a}, *,{ }^{*}}$, Jinshang $\mathrm{Li}^{\mathrm{b}}$, Jie Chen ${ }^{\mathrm{b}}$, Suqing Zhang ${ }^{\mathrm{a}}$, Jin Wang $^{\mathrm{a}}$, Frank C. Walsh ${ }^{\mathrm{c}}$, Shuncai Wang ${ }^{\mathrm{c}, * *}$, Yanpeng Xue ${ }^{\mathrm{d}}$

${ }^{a}$ Shandong Provincial Key Laboratory for High Strength Lightweight Metallic

Materials, Advanced Materials Institute, Qilu University of Technology (Shandong Academy of Sciences), Jinan, 250014, China

${ }^{b}$ School of Environmental and Chemical Engineering, Shanghai University, Shanghai, 200444, China

${ }^{c}$ National Centre for Advanced Tribology at Southampton (nCATS), University of Southampton, SO17 1BJ, UK

${ }^{d}$ National Center for Materials Service Safety, University of Science and Technology Beijing, Xueyuan Road 30, 100083, Beijing, China

※ J. Zhou and G. Zhao contributed equally.

*zhaogch@sdas.org, ${ }^{* *}$ wangs@ @soton.ac.uk 
Abstract: Weak hierarchical rough structures on superhydrophobic surfaces hinder their practical industrial applications. This paper reports a strategy to fabricate robust nickel-based superhydrophobic coatings via the codeposition of nanosized $\mathrm{WS}_{2}$ and WC particles. The Ni/WC/WS 2 composite coatings were synthesized on mild steel substrates by one-pot electroplating followed by the adsorption of stearic acid selfassembled monolayers to modify the surface wetting. The particle concentration in the bath was investigated and the maximum water contact angle of approx. 170 deg was achieved by optimizing the particle contained. Hardened by WC and lubricated by $\mathrm{WS}_{2}$ inclusions the superhydrophobic coatings showed remarkable abrasive resistance with a bearing capacity $\geq 10000 \mathrm{~mm}$ abrasion length. The coatings also showed aerophilic behavior and good environmental stability over more than 6 months.

Keywords: electroplating; $\mathrm{Ni} / \mathrm{WC} / \mathrm{WS}_{2}$; composite; superhydrophobic; abrasion

\section{INTRODUCTION}

Surface engineering offers enormous potentials on improving the corrosion and wear resistance, enhancing mechanical properties, providing antifouling or antimicrobial properties on the surfaces as well as transforming electronic and aesthetic properties [1]. Coatings, enabling surface robust against wear and tribocorrosion, are the most important part of surface engineering. The functional coatings which possess a combination of the above properties have been extensively sought after in recent years. One of the most characteristics - superhydrophobicity was widely investigated due to wide potential applications such as self-clean, anti-corrosion, antifouling, energy 
conversion and catalysis [2-9]. These advanced materials have also significantly contributed to progress in surface science.

The recent extensive research of superhydrophobic coating was inspired from lotus leaf wetting, known as the "lotus effect" [10]. The water-proofing coatings which has large contact angle with water (at least $150 \mathrm{deg}$ ) and small water sliding angle (maximum $5 \mathrm{deg}$ ) allow water droplets to rapidly roll off the surface instead of wetting. According to the Wenzel-Cassie approach $[11,12]$, superhydrophobicity results from two factors, namely a low interface energy (hydrophobic or aerophilic behavior) and a rough surface (hierarchical micro and nano structures). With a continuous "air cushion" formed and entrapped between solid and liquid phase, the water droplet tends to maintain its shape with high contact angle. A lower energy surface facilitates rapid water roll off or rebound, maintaining the falling droplet [13].

Currently, the most common problem for superhydrophobic coatings is lack of a compact, dense structure for wear resistance [14]. Superhydrophobicity can be diminished quickly if the hierarchical micro and nano structures are worn by surface abrasion, which is a main challenge in practical applications. Work to improve abrasion resistance in recent years has focused on fluorine-containing chemicals such as (heptadecafluoro-1,1,2,2-tetradecyl) triethoxysilane modified nickel coating [15], fluorooxysilane [16], perfluorooctyltriethoxysilane modified silica-based coatings [17], and also includes using fluoro-free chemicals like micro-porous PDMS [18], organosilanes [19]. Such coatings showed excellent superhydrophobicity and abrasive resistance, which presented potential usage in many applications. In our previous study, 
we successfully fabricated a Ni-WS 2 superhydrophobic coating which had a contact angle of $158.3 \mathrm{deg}$ and a sliding angle of $7.7 \mathrm{deg}$ [20]. The coating showed positive shifted corrosion potential at $-0.162 \mathrm{~V} v s$. SCE and low corrosion current density about $2.07 \times 10^{-7} \mathrm{~A} \mathrm{~cm}^{-2}$, and excellent self-cleaning property. However, because of its porous structures, the rough surface showed a limited abrasive resistance to hold its superhydrophobic. The physical modelling proved that the porous coating possesses a lower hardness than a dense one [20a] where the wear rate is proportional to hardness according to the Archard's law. Thus, a compact coating is beneficial to enhance wear resistance properties, which are main concerns for most superhydrophobic coatings.

The fabrication of composite coatings with inclusion of single-phase particles is well established. The co-deposition of the varied inclusions with different physical and properties has been anticipated more effective in practice [21]. Such particles are either soft and lubricating, e.g. PTFE or C or hard and wear resistant, e.g. SiC, WC. Use of nanosized particles may further control the diverse surfaces of coatings [22]. It is important to consider combining different types of particles, e.g. soft and hard, lubricating and wear resistant [23], but few have been reported.

The roughness and compactness of composite electrodeposits could be improved by ultrasonic assistance [24], high shear mixing [25], adding refiner [20] and alloying [26]. In this study, we propose a strategy to fabricate coatings with robust superhydrophobic surface, by co-deposition of $\mathrm{Ni}-\mathrm{WC}-\mathrm{WS}_{2}$ composite followed by an organic modification. $\mathrm{WS}_{2}$ nanoparticles $\left(\mathrm{WS}_{2} \mathrm{NPs}\right.$ ) as solid self-lubricant can significantly reduce the coefficients of friction between solid-solid contacts [20, 26]. WC 
nanoparticles (WC NPs) are hard materials to increase the abrasive resistance [23]. The combination of these two types of particles ensures maximum anti-abrasion. Electroplating is well known as a facile, cost-effective method to fabricating coatings. Stearic acid as the surface energy modification agent has the advantages of (a) environmental friendly and low-cost properties compared with fluorine-containing chemicals, (b) strong absorption on the surface of nickel (as the $\mathrm{C}-\mathrm{O}-\mathrm{Ni} \mathrm{i}_{(111)}$ bond formed bidentate interaction [27]). Based on the above design we successfully prepared a robust $\mathrm{Ni}-\mathrm{WC}-\mathrm{WS}_{2}$ coating showing strong superhydrophobicity (maximum contact angle up to $170 \mathrm{deg}$ and sliding angle as low as $0 \mathrm{deg}$ ), abrasive resistance (10000 $\mathrm{mm}$ abrasion length) and long-term stability (at least 6 months). We believe that this approach could provide a crucial step in bringing robust superhydrophobic surfaces to practical engineering applications.

\section{EXPERIMENTAL DETAILS}

\subsection{Materials and coating deposition}

AISI mild steel was used as substrate and machined to the dimensions of $90 \mathrm{~mm} \times$ $10 \mathrm{~mm} \times 3 \mathrm{~mm}$, prepared as cathode. The substrate (i.e. cathode) was low hardenability and low tensile AISI 1020 mild steel with Brinell hardness of 119-235 and tensile strength of 410-790 MPa. This steel responds well to cold work and heat treating. Weldability is fair and mainly used as shafts, gears, pins and chains etc. Nickel plate with the same dimensions was used as anode. All the electrodes were polished with 240 and 800 grit $\mathrm{SiC}$ sandpaper. The electroplating bath was based on Watts Bath, with the addition of hexadecyl trimethyl ammonium bromide $\left(\mathrm{CTAB}, \mathrm{CH}_{3}\left(\mathrm{CH}_{2}\right)_{15} \mathrm{~N}(\mathrm{Br})\left(\mathrm{CH}_{3}\right)_{3}\right.$, 
99\%, Shanghai Aladdin Bio-Chem Technology Co., Ltd), WS 2 NPs (200 nm, Shanghai ST-NANO Science \& Technology Co., Ltd) and WC NPs (400 nm, Aladdin). The concentration range of components can be found in Table 1. Ultrasonic cleaner was deployed for 20 minutes to assist the dispersion of nanoparticles in bath before use.

The chemical compositions of substrate, anode and nanoparticles were analyzed by EDS. As shown in Fig. S1, the mild steel plate is composited by $\mathrm{Fe}, \mathrm{Mn}, \mathrm{Al}, \mathrm{C}$ with a Fe content of 97.7 wt.\%. 98.0 wt.\% nickel plate was used as anode. The $\mathrm{WC}$ and $\mathrm{WS}_{2}$ NPs have little $\mathrm{O}$ element as impurity was probably resulted from the fabrication process of particles. Nevertheless, the atomic ratio of $\mathrm{S}$ and $\mathrm{W}$ in $\mathrm{WS}_{2} \mathrm{NPs}$ is equal to1.86:1, while this value of $\mathrm{C}: \mathrm{W}$ in WC NPs is calculated as $1.14: 1$, which are generally consistent with the theoretical values.

Before electroplating, the electrodes were rinsed in distilled water and immersed in hydrochloric acid for 10 seconds to remove oxides and organic films on the surface, then washed with acetone and distilled water. The electrodes were covered by polyester tape leaving an area of $4 \mathrm{~cm} \times 1 \mathrm{~cm}$ exposed for deposition. In the two-electrode cell, the electrodes were parallel to each other held by an electrode holder. A cylindrical PTFE-coated steel magnetic follower of $6 \mathrm{~mm}$ diameter and $25 \mathrm{~mm}$ length was applied for bath agitation. The electroplating parameters were as following: current density of $4 \mathrm{~A} \mathrm{dm}^{-2}$, temperature of $40^{\circ} \mathrm{C}$ and stirring speed of $600 \mathrm{rpm}$ over a plating time of 30 minutes.

After electroplating, the fabricated Ni-WC-WS 2 coating was sufficiently cleaned by distilled water and acetone, followed by ultrasonic agitation $(59 \mathrm{kHz}, 100 \mathrm{~W})$ for 30 
minutes in $2 \mathrm{mM}$ stearic acid - acetone solution. The sample was then dried in vacuum at room temperature $\left(20^{\circ} \mathrm{C}\right)$ for 3 hours.

\subsection{Characterization of the coatings}

The surface morphologies of the coatings were investigated by scanning electron microscopy (SEM, ZEISS, EVOMA10). The composition and elements mapping were determined using an energy dispersive spectrometer (EDS, Oxford) in the SEM mode. 3D surfaces were constructed by Alicona 3DMeX surface profilometer analysis. The wettability of the coating surface including water contact angle and sliding angle was analyzed by an optical contact angle measuring system (EASTERNDATAPHY OCA 15EC). The volume of water droplet was $6 \mu \mathrm{L}$. The angles were measured by DropSnake which is a plugin of ImageJ (software) to shape the drop. Surface roughness was measured by a surface roughness tester (TR221, TIME Group Inc.). X-ray diffraction (XRD) experiments were performed on X'Pert POWDER. The images in long-term stability test were captured by a digital camera (12-megapixel) and the videos were taken at 240 frames per second.

The linear abrasion test was carried out to evaluate abrasive resistance of superhydrophobic Coating 15-5. In the test, the 360\# grit aluminum oxide paper was horizontally placed on a smooth laboratory bench, which was selected as the abradant. After that, put the sample face-down to sandpaper, with the plane contact, as shown in Video S2. $100 \mathrm{~g}$ weight was place on the back of sample. Considering the weight of sample $(20.67 \mathrm{~g})$, the total counter weight was calculated as $120.67 \mathrm{~g}$, which provided vertical pressure of $\sim 3 \mathrm{kPa}(2.956 \mathrm{kPa})$. The coating faced to sandpaper and moved 
along ruler for $100 \mathrm{~mm}$ as one cycle. The total abrasion length was $14,000 \mathrm{~mm}$ (i.e. 140 cycles). The contact angle was measured after each cycle in the first $1000 \mathrm{~mm}$ abrasion, and each 5 cycles for the remaining.

\section{RESULTS AND DISCUSSION}

\subsection{Electrodepositing arrangement and mechanism}

Fig. 1a and Fig. 1b show the set-up and mechanism involved in the deposition of superhydrophobic composite coatings. As can be seen in Fig. 1a, the arrangement consists of four components, namely, the electrodes, cell, heater and stirrer. The planar nickel anode provides nickel ions to maintain a constant concentration of $\mathrm{Ni}^{2+}$. Nickel and particles were co-deposited on the mild steel cathode surface. The electrode holder, which was designed and 3D printed, was mounted on the top of beaker to secure the cathode and anode. The interelectrode gap was fixed at $30 \mathrm{~mm}$. The electrolyte was the Watts bath mentioned above. The temperature of the bath was measured by a thermometer and controlled $40 \sim 42{ }^{\circ} \mathrm{C}$ by a circulating water bath. The solution was continuously agitated by magnetic stirring.

A key factor in realizing micro/nano hierarchical is to make optimum use of the surfactant and nanoparticles. CTAB is a cationic surfactant. Positive charges are conferred to the nanoparticle surface by adsorption, as shown in the inset of Fig. 1b. In this study, after prolonged agitation and ultrasonic dispersion, the surfaces of WC NPs and $\mathrm{WS}_{2}$ NPs were mostly covered by CTAB forming cationic particles. Under the effect of electric field, WC NPs and $\mathrm{WS}_{2}$ NPs migrated to the cathode, adsorbed, then co-deposited into the growing nickel matrix. The primary reactions at the cathode and 
the anode are shown below as equation (1) and (2). Two kinds of particles were codeposited for which different growth rates contributed to the micro/nano rough surface. The WC NPs and $\mathrm{WS}_{2}$ NPs improved abrasive resistance of coatings; the combined effects are discussed below. The mechanism on one type of particle has been considered in our previous studies $[20,25,26,28,29]$. In addition, the current density of electrodeposition was fixed at $4 \mathrm{~A} \mathrm{dm}^{-2}$ to avoid the hydrogen evolution reaction (equation (3)) at anode which was harmful to coating.

$$
\begin{array}{ll}
\text { Equation (1): } & N i^{2+}+2 e^{-} \rightarrow N i \\
\text { Equation (2): } & N i \rightarrow N i^{2+}+2 e^{-} \\
\text {Equation (3): } & 2 H^{+}+2 e^{-} \rightarrow H_{2}
\end{array}
$$

\subsection{Surface morphologies, roughness and wettability}

According to the Wenzel approach, the wettability of the surface can be enhanced if its roughness increases [11]. Hydrophobicity would follow the same principle. Fig. 2 shows SEM images of selected samples. The samples were fabricated from a bath containing $5 \mathrm{~g} \mathrm{dm}^{-3} \mathrm{WS}_{2}$ NPs (Fig. 2a, 0-5), $10 \mathrm{~g} \mathrm{dm}^{-3}$ WC NPs (Fig. 2b, 10-0), $20 \mathrm{~g}$ $\mathrm{dm}^{-3} \mathrm{WS}_{2}$ NPs and $5 \mathrm{~g} \mathrm{dm}^{-3} \mathrm{WC}$ NPs (Fig. 2c, 20-5), $10 \mathrm{~g} \mathrm{dm}^{-3} \mathrm{WS}_{2} \mathrm{NPs}$ and $15 \mathrm{~g} \mathrm{dm}^{-}$ ${ }^{3}$ WC NPs (Fig. 2d, 10-15). The only $\mathrm{WS}_{2}$ nickel deposit, as shown in Fig. 2a, has large and irregularly clusters randomly located on the surface of the coating. The lengths of clusters were measured (Fig. 2a-i) maximum up to $56.79 \mu \mathrm{m}$, minimum as low as 21.44 $\mu \mathrm{m}$. The Coating 10-0 was fabricated from $10 \mathrm{~g} \mathrm{dm}^{-3}$ WC NPs in the nickel bath. Clusters still emerged on the surface, but they seem regularly shaped and have more uniform distribution than 0-5, as can be seen in Fig. 2b \& 2b-i (about $43.24 \mu \mathrm{m}$ in 
length). Dramatically, the clusters have disappeared in Coatings 20-5 and 10-15. Fig. $2 \mathrm{c} \& 2 \mathrm{~d}$ show that the coatings fabricated from $\mathrm{WS}_{2} \& \mathrm{WC}$ have more compact, fine surface morphologies than the other two, which indicates $\mathrm{WS}_{2}$ NPs and WC NPs contribute to refining surface structures. Just like demonstrated by Fig. S2 and Fig. S3 in supplementary, this phenomenon is not incident: as dissimilar particle gradually added in bath, the surface structures are refined step by step.

Fig. 3 illustrates the growth mechanism of rough surface. Since nanosized particles, such as $\mathrm{WS}_{2}$ and $\mathrm{WC}$, are electrically conductive, the nickel ions can be reduced both on the nickel deposits and on the surface of nanoparticles. This growth mechanism has been investigated and described in the previous studies, such as $\mathrm{Co} / \mathrm{WS}_{2}[30], \mathrm{Ni} / \mathrm{WS}_{2}$ [20, 24], Ni/WC [29, 31], Ni/P/WS 2 [26] and Ni/MoS 2 [25]. In this study, we found the surface morphology closely depended on the type and concentration of nanoparticles. As showed in Fig. 3, the CTAB capped particles and $\mathrm{Ni}^{2+}$ transports to electrical double layer from bulk solution by both convective-diffusion and electrophoretic migration. Because of the differences in electrical resistivity of nanoparticles and nickel matrix, the distribution of surface potential is irregular once particles touch the surface of cathode. The electrical resistivities of nickel $\left(\rho_{\mathrm{Nickel}}\right), \mathrm{WC}\left(\rho_{\mathrm{WC}}\right)$ and $\mathrm{WS}_{2}\left(\rho_{\mathrm{WS}_{2}(a b)}\right.$, are $69.3 \mathrm{n} \Omega \mathrm{m}, 200 \mathrm{n} \Omega \mathrm{m}$ and $2.4 \times 10^{8} \mathrm{n} \Omega \mathrm{m}$ (parallel to $c$ plane, higher in perpendicular [32]), respectively. Fig. $2 \mathrm{a} \& 2 \mathrm{~b}$ show the different surface morphologies of $\mathrm{Ni} / \mathrm{WS}_{2}$ and $\mathrm{Ni} / \mathrm{WC}$ coatings. Considering much higher $\rho_{\mathrm{WS}_{2}}$ than $\rho_{\text {Nickel }}$, the current distribution around $\mathrm{WS}_{2}$ particles attached on the cathode is changed significantly. The intensity is much stronger than that caused by WC, since WC NPs has approximately 
the same electrical resistance as the nickel matrix. Thus, a higher current density is formed on $\mathrm{WS}_{2}$ NPs. After long term deposition, clusters are generated on Coating 05, and the coating has more rough structures than $\mathrm{Ni} / \mathrm{WC}(10-0)$. The refining mechanism of Ni/WC/WS 2 coating is complicated (Fig. 2c \& 2d), and will study in the further. Probably, nickel ions, WC NPs, $\mathrm{WS}_{2}$ NPs are deposited on the surface of each other and subsequently the rough structures are combined together, as in the crosslinking effect in a hydrogel.

Although the samples obviously produced different surface morphologies, the distinction in wettability after modified by stearic acid was not significant. Images of water contact angles in Fig. 2 demonstrate all the samples as superhydrophobic. The measurements of contact angles are listed in Table 2. The data (statistical average) are distributed between $160 \mathrm{deg}$ and $170 \mathrm{deg}$. Among them, the minimum is $155.3 \mathrm{deg}$ on the surface of Coating 0-5, and maximum are 170.4 deg of Coatings 10-15 and 171.0 deg of Coating 20-5. The differences in morphologies do not have much contribution to contact angles. As smooth surface has stronger abrasive resistance than rough surface, the refining effect of these two nanosized particles will be beneficial to fabricate robust superhydrophobic surface by electroplating.

In order to analyze the surface roughness and demonstrate the effect of refining by nanoparticles, the 3D SEM images and stereoscopic images of Coatings $0-5,10-0$ and 10-15 were constructed by Alicona 3DMeX, as seen in Fig. 4. Irregularly located clusters are visible on the surface of Coating 0-5 (Fig. 4a). The clusters are not uniform with different heights (Fig. 4a-i). The morphologies of clusters are improved on Coating 
10-0 in Fig. 4 b \& b-i. For Coating 10-15, there is no apparent cluster observed and the surface shows a smooth feature. The parameters of surface roughness of the three samples are listed in Table $3 . \mathrm{S}_{\mathrm{a}}$ (arithmetical mean height) of Coating 10-15 are obvious smaller than that of $0-5$ and $10-0$, which demonstrates that the co-deposition of these two kinds particles contribute to fabricate smooth surface without big clusters. Coating 10-15 has the smallest maximum height $\left(\mathrm{S}_{\mathrm{z}}\right)$ among the three coatings, which means $10-15$ is mechanically stable since high structure is easy to fragment from the root under external force. The value of skewness $\left(\mathrm{S}_{\mathrm{sk}}\right)$ represents the degree of bias of the roughness shape. Coating 10-15 had smallest absolute $\mathrm{S}_{\mathrm{sk}}$ and largest contact angle among the coatings as a symmetrical height distribution $\left(\mathrm{R}_{\mathrm{sk}}\right.$ close to 0$)$ is expected to enhance superhydrophobicity [26, 33]. Finally, the height distribution of 10-15 is normal according to the value of $S_{\mathrm{ku}}$ (close to 3), while 0-5 is skewed $\left(\mathrm{S}_{\mathrm{ku}}<3\right)$ and $10-0$ is spiked $\left(\mathrm{S}_{\mathrm{ku}}>3\right)$. From the discussion of the surface parameters above, it suggests that $\mathrm{Ni} / \mathrm{WS}_{2}$ and $\mathrm{Ni} / \mathrm{WC}$ tend to grow sideways and lengthways, respectively. The synergistic effect of $\mathrm{WS}_{2}$ NPs and WC NPs in electrodeposition contributes to a fine surface morphology with little cluster of Coating 10-15.

The roughness average $\left(\mathrm{R}_{\mathrm{a}}\right)$, as the most commonly used surface parameter, was applied to investigate the effects of nanoparticles on surface profiles. $R_{a}$ of all the asprepared composite coatings were measured by stylus tracing method with a measurement length of $1.25 \mathrm{~mm}$. The results were statistically analyzed as 3D surface and contour using Design-Expert (software) and shown in Fig. 5. From the analysis of variance table, the model F-value of 7.67 is significant. The value of "Prob $>F$ " of WC, 
$\mathrm{WS}_{2}$ and $\mathrm{WC} \cdot \mathrm{WS}_{2}$ (WC and $\mathrm{WS}_{2}$ interaction) are $0.003,0.0172$ and 0.0012 , respectively, which can be decided as significant model terms. From Fig. 5a, it can be directly found that the deposition of single particle increases the surface roughness dramatically, from $0.653 \mu \mathrm{m}$ to $20.047 \mu \mathrm{m}$ for $\mathrm{WS}_{2}\left(0-20 \mathrm{~g} \mathrm{dm}^{-3}\right)$, and from $0.653 \mu \mathrm{m}$ to $8.059 \mu \mathrm{m}$ for $\mathrm{WC}\left(0-20 \mathrm{~g} \mathrm{dm}^{-3}\right)$. According to the analysis of this figure, there are two conditions for the $\mathrm{R}_{\mathrm{a}}$ variation. At low concentration of $\mathrm{WC}\left(<8.378 \mathrm{~g} \mathrm{dm}^{-3}\right)$ and $\mathrm{WS}_{2}\left(<4.595 \mathrm{~g} \mathrm{dm}^{-3}\right), \mathrm{R}_{\mathrm{a}}$ increases with the inclusion of second phase particle. On the contrary, at high concentration of particles, $\mathrm{R}_{\mathrm{a}}$ is reduced by increasing concentration of second phase particle. Roughness in examples given in Fig. 5b (Curve I \& II) show downtrends with the inclusion of different particle. In curve I, as $\mathrm{WS}_{2}$ concentration fixes at $5 \mathrm{~g} \mathrm{dm}^{-3}, \mathrm{R}_{\mathrm{a}}$ decreases from $4.366 \mu \mathrm{m}$ to $1.892 \mu \mathrm{m}$ with increasing WC concentration. Similarly, $\mathrm{R}_{\mathrm{a}}$ also can be reduced by $\mathrm{WS}_{2}$ when $\mathrm{WC}$ concentration is fixed at $10 \mathrm{~g} \mathrm{dm}^{-3}$, as shown in curve II. These phenomena are in agreement with the analysis results, which can be referred for further electroplating.

The experiments of wire cutting and polishing were carried out to prepare the sample for cross-section observation, which were used to evaluate the compactness of superhydrophobic coatings. In order to eliminate the effect of HAZ, careful polishing was carried out before observation. As demonstrated by Fig. S4, $\sim 1 \mathrm{~mm}$ depth of polishing was enough to neglect the effect of HAZ. Fig. 6 shows the cross sections of Coating 0-5, 10-0, 10-15. A loose and irregular coating can be found on the surface of Coating 0-5 (Fig. 6a), while Coating 10-0 (Fig. 6b) exhibits more uniform but porous. However, the layer on Coating 10-0 is not much close to the mild steel substrate. Under 
the same electrodeposition conditions, a compact layer without void close to the mild steel substrate can be found for $\mathrm{Ni} / \mathrm{WC} / \mathrm{WS}_{2}$ composite coating (Fig. 6c), which demonstrates the co-depositing of $\mathrm{WC}$ and $\mathrm{WS}_{2}$ nanosized particles refine the surface structures and improve the adhesion of coating.

\subsection{Surface element analysis and wettability}

To investigate chemical composition on the surface of coatings, EDS test was carried out. The area selected for EDS analysis was $35200 \mu \mathrm{m}^{2}(220 \mu \mathrm{m} \times 160 \mu \mathrm{m}$, full screen $)$, magnification was $\times 500$. The percentage of $S$ was used to calculate the $\mathrm{WS}_{2}$ content and the $\mathrm{WC} \%$ was obtained by remain W. Moreover, the particles' structures in coating were further study by XRD analysis in the following section. In order to enhance believable degree of EDS results, we prolonged the live time to detect more counts. As shown by Fig. R2, the counts are 200,196, then the relative error can be calculated as $0.22 \%(1 / \sqrt[2]{200196})$

Fig. $7 \mathrm{a}$ and Fig. $7 \mathrm{~b}$ show the content of $\mathrm{WC}$ and $\mathrm{WS}_{2}$ on the coatings fabricated against their concentration in bath. Initially there is an increase in the WC particle content with the bath loading of particles, maximum at 23.4 wt.\% (Coating 10-5). After that, a downward trend can be seen. In comparison, the content of $\mathrm{WS}_{2}$ is slightly decreased from $5.22 \mathrm{wt} . \%$ to $0.69 \mathrm{wt} . \%$. As the concentration of WC fixed at $10 \mathrm{~g} \mathrm{dm}^{-}$

${ }^{3}$, showed by Fig. $7 b$, the addition of $\mathrm{WS}_{2}$ NPs in bath effectively enhances the deposition of $\mathrm{WS}_{2}$. Then the content of $\mathrm{WS}_{2}$ keep stable though a slight reduction can be witnessed after the concentration bigger than $10 \mathrm{~g} \mathrm{dm}^{-3}$. The content of WC in Fig. 
$7 \mathrm{~b}$ has a similar change with Fig. 7a. In our opinion, the phenomenon can be explained by considering three aspects:

(i) The two particles are competitive for their transportation to the cathode during electroplating. WC NPs are hydrophilic, whereas $\mathrm{WS}_{2}$ NPs are hydrophobic [20]. The charged WC NPs are much more flexible in the diffusion process than $\mathrm{WS}_{2}$ NPs, which results plentiful WC NPs diffuse to electrical double layer and deposit in coating by purely mechanical entrapment. This contributes to high $\mathrm{WC}$ and poor $\mathrm{WS}_{2}$ in coatings.

(ii) A maximum particle content is achieved when a saturation point is reached [23].

(iii) Since no further surfactant added $\left(\mathrm{CTAB}=0.1 \mathrm{~g} \mathrm{dm}^{-3}\right)$, high concentration of nanosized particles in bath causes agglomeration, especially for the hydrophobic $\mathrm{WS}_{2}$ NPs in aqueous solution, which is harmful for the incorporation of particles.

Surface energy of WC is $1.7 \mathrm{~J} \mathrm{~m}^{-2}$ [34], which is about 25 times higher than that of $\mathrm{WS}_{2}$ (in the range $0.065-0.075 \mathrm{~J} \mathrm{~m}^{-2}$ [35]). The calculated value of surface energy is $20.35 \mathrm{~J} \mathrm{~m}^{-2}$ for plane (111) of nickel deposits [36]. As showed by Fig. 7a \&7b, all the $\mathrm{Ni} / \mathrm{WC} / \mathrm{WS}_{2}$ coatings have little $\mathrm{WS}_{2}$ content on surfaces. Because of the high surface energy of $\mathrm{Ni}$ and $\mathrm{WC}$, the coatings are hydrophilic or even superhydrophilic on the rough surface, as the experiment demonstrating. Video S1 shows the superhydrophilicity on the surface of dry Coating 10-5. After modified by stearic acid, the samples' wettability was switched from (super) hydrophilic to superhydrophobic. Fig. $7 \mathrm{c} \& 7 \mathrm{~d}$ reveal the hydrophobicity affected by the content of WC \& $\mathrm{WS}_{2}$ respectively. As can be seen from Fig. 7c, firstly the contact angles increase with WC content since proper hierarchical morphology is forming. Then high WC content results 
to high surface energy, which contributes to a slight decrease of contact angles. For $\mathrm{WS}_{2}$ content in Fig. 7d, the contact angles remain high, fluctuating between $160 \mathrm{deg}$ and 170 deg, indicating $\mathrm{WS}_{2}$ have little effects on wettability after modification.

The EDS element maps on the surface of coating reflect their nucleus and growth during electroplating process. Coating 15-5 was taken for the elemental mapping. The selected area was about $988 \mu \mathrm{m}^{2}$. Fig. 8 shows the distribution of nickel (Ni), tungsten (W) and sulfur (S). The map of element $\mathrm{S}$ represents the position of $\mathrm{WS}_{2} \mathrm{NPs}$ while the map of $\mathrm{W}$ is from both $\mathrm{WC}$ and $\mathrm{WS}_{2}$. It shows that nickel cover both smooth area and the hierarchical micro-nano structures. The nanosized particles prefer to deposit on the top of the lotus-like clusters. This is coincident with the analysis of electroplating mechanism in Fig. 3: high current density occurred surrounding the nanosized particles offer an opportunity of priority growth. Therefore, the hierarchical structures are formed after the long-time deposition.

\subsection{Orientations of coatings}

The crystal planes of coatings were investigated by X-ray diffraction. Fig. 9 shows the XRD patterns of the selected samples. All the peaks are indexed referring to standard Powder Diffraction Files, i.e. JCPDS 04-0850 for nickel, JCPDS 84-1398 for $\mathrm{WS}_{2}$ and JCPDS 72-0097 for WC. As shown in Fig. 9a, the intensity of peaks diffracted by nickel crystals on pure nickel deposits are relatively sharper and stronger than the other samples, which indicates the pure nickel coating may have large crystallite size. According to Debye-Scherrer equation, the crystallite sizes of nickel deposits are calculated as $56.2 \mathrm{~nm}$ for (111) and $80.9 \mathrm{~nm}$ for (200). The strongest peak on (200) 
indicates the minimization of elastic strain energy is the driving force for the pure $\mathrm{Ni}$ coating. The crystallite sizes of coating were reduced by the incorporation of $\mathrm{WS}_{2} \mathrm{NPs}$ and WC NPs. For instance, nickel crystallite sizes of Coating 20-5 are $31.0 \mathrm{~nm}$ of (111) and $22.1 \mathrm{~nm}$ of (200). All the results of crystallite sizes calculated from Fig. S5 \& S6 are listed in Table 4. With the incorporation of the nanosized particles, the preferred orientation of nickel crystal in composite coating is changed from (200) to (111). The similar phenomena were also observed in $\mathrm{Ni} / \mathrm{WS}_{2}$ [24], $\mathrm{Ni} / \mathrm{WC}$ [29] and $\mathrm{Ni} / \mathrm{Al}_{2} \mathrm{O}_{3}$ [37]. The dominated (111) plane of nickel crystal is reported to minimize the surface energy [36], The presence of strongest (111) $)_{\mathrm{Ni}}$ peaks indicates the surface energy will decrease with the plating progress. This also proves that the particles help to fabricate robust superhydrophobic coatings. The strong absorption of stearic acid (low surface energy modifier) by C-O-Ni bonds will also enhance this effect [27].

Fig. $9 \mathrm{~b}$ shows the details of XRD pattern on Coating 10-15, with Miller index. The peaks of (111) and (200) belong to fcc nickel. The corresponding (002), (004), (100), (101), (103), (110) confirm hcp $\mathrm{WS}_{2}$, while (001), (100), (101), (110), (111) are consistent with the hcp WC.

\subsection{Abrasive resistance}

Fig. 10 shows the results of abrasion test on the Coating 15-5. In Fig. 10, the variation tendency of contact angles can be divided into three stages, fluctuated decline in the primary stage (abrasion length in the range of $0 \mathrm{~mm}-2000 \mathrm{~mm}$ ), kept in stable in the secondary stage $(2000 \mathrm{~mm}-10000 \mathrm{~mm})$, and sharp decrease in the tertiary stage (10000 $\mathrm{mm}$ to the end). In the primary stage, the contact angels slightly decrease from about 
$166 \mathrm{deg}$ initially to $160 \mathrm{deg}$ at $2000 \mathrm{~mm}$ length, as shown in Fig. 10b. In the early runin period, the surfaces of coating and paper adapt to each other results in a minor damage to the as-prepared hierarchical rough structures of coating. Afterwards, the contact angle remains in the range of $153 \mathrm{deg}$ to $156 \mathrm{deg}$. This stage is relatively longlasting since a stable tribofilm is formed by the self-lubricating $\mathrm{WS}_{2}$ NPs (Fig. 10c). Gradual exfoliation of the inorganic fullerene-like onions and transfer of monomolecular $\mathrm{WS}_{2}$ sheets onto the metal surface is the major role to alleviate friction and wear [38]. The coefficient of friction of nickel based $\mathrm{WS}_{2}$ composite coating could be as low as 0.17 in our previous study [26]. The self-lubricating effect is failed and $\mathrm{WS}_{2}$ NPs lose function started from $10000 \mathrm{~mm}$ abrasion length with a dramatical decrease in contact angles. Nanostructure is essential for superhydrophobic surfaces with high contact angle, while the hierarchical structures contribute to low droplet sliding angle [39]. We believe the hierarchical structures are worn in this stage, as illustrated in Fig. 10d.

SEM images and EDS test were analyzed in order to investigate the failure in superhydrophobicity of the coating after abrasion. Fig. 11 shows the surface morphology after abrasion. As can be seen in Fig. 11a, at low magnification the surface shows smooth but porous morphology. Several nonconducting particles (black area indicated by arrow) appears, which is characterized as aluminum oxide by EDS spectra $(\mathrm{Al} \mathrm{K}=1.486 \mathrm{keV}$ ). The particles have the size in the range of $30-40 \mu \mathrm{m}$, which can be confirmed as separated from the abrasive paper and absorbed on the coating. In addition, some abrasive traces are found on the surface. Fig. $11 \mathrm{~b}$ is the SEM image in 
high magnification. After abrasion, the top parts of hierarchical structures are worn and removed by the polish powders, which is in agreement with the mechanism illustrated by Fig. 10d. The feature is typical morphology of abrasive wear as the scratch can be clearly seen.

\subsection{Long-term stability and aerophilic behavior}

Long-term stability is one of the key indexes to evaluate the quality of superhydrophobic coating. Fig. 12 shows the 6-month stability test of Coating 10-15. The coating exhibits uniform surface and dark color in air presented by the optical photograph in Fig. 12a. By injecting pure water into the beaker, a shining layer is formed on the surface of coating, with clear boundary (Fig. 12b). This is so called "air cushion" anchored by the superhydrophobic coating. A great deal of air is captured and anchored in the gap between micro/nano structures and an extremely small area of coating contacts with water. According to the Cassie-Baxter equation [12], the solidwater contact area only accounts for $2.60 \%$ of the solid surface, i.e. $97.40 \%$ of the coating surface is in contact with air. After the 6 months standing at room temperature $\left(18 \sim 24^{\circ} \mathrm{C}\right)$, the substrate of sample has been corroded, as seen in Fig. 12c; brown rust is formed on the mild steel substrate. There is no color change on the coating (Fig. 12c) and the air cushion is still obviously preserved after immersing the sample into water, indicating that the as-prepared coating is able to retain its superhydrophobicity for at least 6 months.

In order to characterize the long-term stability of the superhydrophobic coating, the surface morphologies and chemical composition were studied by SEM and EDS, as 
shown in Fig. 12a \& 12c. The surface morphology of rust is scaly and porous which is not compact and cannot hinder further corrosion. Showed by EDS analysis, the rust is mainly composed by the elements of $\mathrm{Fe}, \mathrm{O}$. A spot of elements such as $\mathrm{Cl}, \mathrm{Ca}, \mathrm{Na}$ also can be detected in the corroded area which is from the tap water. For the coating, there is no obvious change in surface morphology. However, the coating is slightly oxidized as the content of $\mathrm{O}$ is increased from $1.36 \mathrm{wt} . \%$ to $2.73 \mathrm{wt} . \%$, showed by EDS analysis in Fig. 12c. But a stable air cushion still can be observed on the surface of coating. The contact angle is decreased from 168.8 deg initially to 157.8 deg finally. Such change in hydrophobicity is caused by the oxidation of nickel coating. Nevertheless, the superhydrophobic coating still shows strong water repellent property.

The long-term stability is affected by the capability of gas absorption on the superhydrophobic surface. Fig. 13 shows the underwater gas bubble adhesion on the surface of Coating 10-15. The surface exhibits a rapid absorption of air bubble once the bubble contacting solid surface (Fig. 13a \& 10b). A large bubble (Fig. 13e) of diameter of $9.5 \mathrm{~mm}$ separates from the surface after several air injections. Then, the air cushion still adhered to the surface following another cycle of injection (Fig. $13 \mathrm{f}-\mathrm{h}$ ). The whole process can be seen in Video S3. The water contact angle is measured as $171.0 \mathrm{deg}$ while the air bubble contact angle is around 15.0 deg in Fig. 14. The sample shows a strong aerophilic behavior, which contributes to preservation of a stable, long-term air cushion on the surface.

\section{CONCLUSIONS}


The Ni/WC/WS 2 composite coatings were fabricated successfully on mild steel substrate by a simple one-pot electroplating followed by fluorine-free surface modification. The results are highlighted below:

a) The $\mathrm{Ni} / \mathrm{WC} / \mathrm{WS}_{2}$ composite coating achieved a smooth surface, regular rough structure without presence of clusters, which was resulted from the synergistic effect of two types of particles. It also possessed a dense and high superhydrophobicity after surface modification with water contact angles up to $170 \mathrm{deg}$. The cross-section revealed inclusion of both WC NPs and $\mathrm{WS}_{2}$ NPs contributed to fabricate compact coating.

b) The particles tended to deposited on the top of roughness structures. The WC NPs deposited faster than $\mathrm{WS}_{2}$ NPs, and the percentage of WC NPs mainly affected the superhydrophobicity of coating.

c) XRD analysis conformed fcc nickel, hcp WC and hcp $\mathrm{WS}_{2}$ deposited on the surface. With the incorporation of WC NPs and $\mathrm{WS}_{2} \mathrm{Nps}$, the orientation preference of nickel deposit was changed from (200) to (111), and the crystallite size of nickel was reduced for 2-4 times.

d)Abrasive test and long-term stability test showed the superhydrophobic $\mathrm{Ni} / \mathrm{WC} / \mathrm{WS}_{2}$ coating obtained remarkable abrasive resistance with a bearing capacity $\geq$ $10000 \mathrm{~mm}$ abrasion length and at least 6 months stability with strong aerophilic behaviour. With the lubricative effect of $\mathrm{WS}_{2}$ NPs, the distance holding superhydropicity for $\mathrm{Ni} / \mathrm{WS}_{2} / \mathrm{WC}$ coating increased 2.5 times than $\mathrm{Ni} / \mathrm{WC}$ coating.

\section{SUPPORTING INFORMATION}


The supplementary material is available at DOI: $\operatorname{xxxxxxxx}$.

EDS analysis of substrate, nickel anode, $\mathrm{WS}_{2}$ NPs, WC NPs (Figure S1), SEM images of samples fabricated from various electrolytes (Figure S2 \& Figure S3), surface morphologies around cutting area (Figure S4), XRD patterns of coatings (Figure S5 \& Figure S6), superhydrophilicity of coating (Video S1), the method of abrasion test (Video S2), aerophilic test (Video S3).

\section{NOTES}

There are no conflicts to declare.

\section{ACKNOWLEDGMENT}

The authors acknowledge financial support by the Royal Society International Exchanges Scheme (IE151191), the EPSRC Global Challenge Research Fund, National Key Research and Development Plan (2017YFB0103904), Natural Science Foundation of Shandong province (ZR2017LEM001) and Youth Fund of Shandong Academy of Sciences (2019QN0023).

\section{REFERENCES}

[1] J.R. Davis, Surface engineering for corrosion and wear resistance, ASM international, 2001.

[2] Y. Lu, S. Sathasivam, J. Song, C.R. Crick, C.J. Carmalt, I.P. Parkin, Robust selfcleaning surfaces that function when exposed to either air or oil, Science, 347 (2015) $1132-1135$.

[3] Q. Liu, D. Chen, Z. Kang, One-step electrodeposition process to fabricate corrosionresistant superhydrophobic surface on magnesium alloy, ACS Appl. Mater. Interfaces, 
7 (2015) 1859-1867.

[4] J. Li, R. Kang, X. Tang, H. She, Y. Yang, F. Zha, Superhydrophobic meshes that can repel hot water and strong corrosive liquids used for efficient gravity-driven oil/water separation, Nanoscale, 8 (2016) 7638-7645.

[5] C.H. Xue, X.J. Guo, J.Z. Ma, S.T. Jia, Fabrication of robust and antifouling superhydrophobic surfaces via surface-initiated atom transfer radical polymerization, ACS Appl. Mater. Interfaces, 7 (2015) 8251-8259.

[6] L. Zhao, Q. Liu, R. Gao, J. Wang, W. Yang, L. Liu, One-step method for the fabrication of superhydrophobic surface on magnesium alloy and its corrosion protection, antifouling performance, Corros. Sci., 80 (2014) 177-183.

[7] P. Zhang, F.Y. Lv, A review of the recent advances in superhydrophobic surfaces and the emerging energy-related applications, Energy, 82 (2015) 1068-1087.

[8] W. Pengwei, H. Toru, M. Qing'an, W. Qianbin, L. Huan, H. Kazuhito, J. Lei, Highly boosted oxygen reduction reaction activity by tuning the underwater wetting state of the superhydrophobic electrode, Small, 13 (2017) 1601250.

[9] Y.P. Xue, A. Taleb, P. Jegou, Electrodeposition of cobalt films with an oriented fir tree-like morphology with adjustable wetting properties using a self-assembled gold nanoparticle modified HOPG electrode, J. Mater. Chem. A, 1 (2013) 11580-11588.

[10] W. Barthlott, C. Neinhuis, Purity of the sacred lotus, or escape from contamination in biological surfaces, Planta, 202 (1997) 1-8.

[11] R.N. Wenzel, Resistance of solid surfaces to wetting by water, Ind. Eng. Chem., 28 (1936) 988-994. 
[12] A.B.D. Cassie, S. Baxter, Wettability of porous surfaces, Trans. Faraday Soc., 40 (1944) 546-551.

[13] J.C. Bird, R. Dhiman, H.-M. Kwon, K.K. Varanasi, Reducing the contact time of a bouncing drop, Nature, 503 (2013) 385.

[14] M. Sedlaček, B. Podgornik, J. Vižintin, Influence of surface preparation on roughness parameters, friction and wear, Wear, 266 (2009) 482-487.

[15] F. Su, K. Yao, Facile fabrication of superhydrophobic surface with excellent mechanical abrasion and corrosion resistance on copper substrate by a novel method, ACS Appl. Mater. Interfaces, 6 (2014) 8762-8770.

[16] A.M. Emelyanenko, F.M. Shagieva, A.G. Domantovsky, L.B. Boinovich, Nanosecond laser micro- and nanotexturing for the design of a superhydrophobic coating robust against long-term contact with water, cavitation, and abrasion, Appl. Surf. Sci., 332 (2015) 513-517.

[17] Z. Qian, S. Wang, X. Ye, Z. Liu, Z. Wu, Corrosion resistance and wetting properties of silica-based superhydrophobic coatings on AZ31B Mg alloy surfaces, Appl. Surf. Sci., 453 (2018) 1-10.

[18] A. Davis, S. Surdo, G. Caputo, I.S. Bayer, A. Athanassiou, Environmentally benign production of stretchable and robust superhydrophobic silicone monoliths, ACS Appl. Mater. Interfaces, 10 (2018) 2907-2917.

[19] J. Zhang, B. Li, L. Wu, A. Wang, Facile preparation of durable and robust superhydrophobic textiles by dip coating in nanocomposite solution of organosilanes, Chem. Commun., 49 (2013) 11509-11511. 
[20] G. Zhao, Y. Xue, Y. Huang, Y. Ye, F.C. Walsh, J. Chen, S. Wang, One-step electrodeposition of a self-cleaning and corrosion resistant $\mathrm{Ni} / \mathrm{WS}_{2}$ superhydrophobic surface, RSC Adv., 6 (2016) 59104-59112.

[20a] C. Ma, S.C. Wang, R.J.K. Wood, Q. Luo, C.T.J. Low, F.C. Walsh, The hardness of porous nanocrystalline Co-Ni electrodeposits, Met. Mater. Int.Metals and Materials Internation, 19 (2013)1197-1192.

[21] C. Kerr, D. Barker, F. Walsh, J. Archer, The electrodeposition of composite coatings based on metal matrix-included particle deposits, Trans. IMF, 78 (2000) 171178.

[22] C.T.J. Low, R.G.A. Wills, F.C. Walsh, Electrodeposition of composite coatings containing nanoparticles in a metal deposit, Surf. Coat. Technol., 201 (2006) 371-383. [23] F.C. Walsh, C. Ponce de Leon, A review of the electrodeposition of metal matrix composite coatings by inclusion of particles in a metal layer: an established and diversifying technology, Trans. IMF, 92 (2014) 83-98.

[24] E. García-Lecina, I. García-Urrutia, J.A. Díez, J. Fornell, E. Pellicer, J. Sort, Codeposition of inorganic fullerene-like $\mathrm{WS}_{2}$ nanoparticles in an electrodeposited nickel matrix under the influence of ultrasonic agitation, Electrochim. Acta, 114 (2013) $859-867$.

[25] N. Zhou, S. Wang, F.C. Walsh, Effective particle dispersion via high-shear mixing of the electrolyte for electroplating a nickel-molybdenum disulphide composite, Electrochim. Acta, 283 (2018) 568-577.

[26] Y. He, W.T. Sun, S.C. Wang, P.A.S. Reed, F.C. Walsh, An electrodeposited Ni-P- 
$\mathrm{WS}_{2}$ coating with combined super-hydrophobicity and self-lubricating properties, Electrochim. Acta, 245 (2017) 872-882.

[27] S.H. Liang, T. Yu, D.P. Liu, W.X. Wang, Y.P. Wang, X.F. Han, Characterization of stearic acid adsorption on $\mathrm{Ni}(111)$ surface by experimental and first-principles study approach, J. Appl. Phys., 109 (2011) 289-295.

[28] Y. He, S.C. Wang, F.C. Walsh, Y.L. Chiu, P.A.S. Reed, Self-lubricating Ni-P-MoS 2 composite coatings, Surf. Coat. Technol., 307 (2016) 926-934.

[29] G. Zhao, J. Li, Y. Huang, L. Yang, Y. Ye, F.C. Walsh, J. Chen, S. Wang, Robust Ni/WC superhydrophobic surfaces by electrodeposition, RSC Adv., 7 (2017) 4489644903.

[30] D.P. Weston, Y.Q. Zhu, D. Zhang, C. Miller, D.G. Kingerley, C. Carpenter, S.J. Harris, N.J. Weston, Co-electrodeposition of inorganic fullerene (IF-WS 2 ) nanoparticles with cobalt from a gluconate bath with anionic and cationic surfactants, Electrochim. Acta, 56 (2011) 6837-6846.

[31] M. Stroumbouli, P. Gyftou, E.A. Pavlatou, N. Spyrellis, Codeposition of ultrafine WC particles in Ni matrix composite electrocoatings, Surf. Coat. Technol., 195 (2005) $325-332$

[32] A. Pisoni, J. Jacimovic, R. Gaál, B. Náfrádi, H. Berger, Z. Révay, L. Forró, Anisotropic transport properties of tungsten disulfide, Scripta Mater., 114 (2016) 48-50. [33] N.D. Boscher, V. Vaché, P. Carminati, P. Grysan, P. Choquet, A simple and scalable approach towards the preparation of superhydrophobic surfaces - importance of the surface roughness skewness, J. Mater. Chem. A, 2 (2014) 5744-5750. 
[34] L. Erik, S. Wolf-Dieter, Tungsten: properties, chemistry, technology of the element, alloys, and chemical compounds, in, New York: Plenum Publishers, 1999.

[35] S. Shafraniuk, Graphene: fundamentals, devices, and applications, Pan Stanford, 2015.

[36] J.M. Zhang, F. Ma, K.W. Xu, Calculation of the surface energy of fcc metals with modified embedded-atom method, Chinese Physics, 13 (2004) 1082.

[37] S.C. Wang, W.C.J. Wei, Kinetics of electroplating process of nano-sized ceramic particle/Ni composite, Mater. Chem. Phys., 78 (2003) 574-580.

[38] L. Rapoport, N. Fleischer, R. Tenne, Fullerene-like $\mathrm{WS}_{2}$ nanoparticles: superior lubricants for harsh conditions, Adv. Mater., 15 (2003) 651-655.

[39] L. Feng, S. Li, Y. Li, H. Li, L. Zhang, J. Zhai, Y. Song, B. Liu, L. Jiang, D. Zhu, Super-hydrophobic surfaces: from natural to artificial, Adv. Mater., 14 (2002) $1857-$ 1860. 